\title{
Biohostability of Different Suture Materials at Different pH
}

\author{
Siddharth Narayan* \\ Department of Microbiology, India \\ *Corresponding Author: Siddharth Narayan, Department of Microbiology, India.
}

Received: July 31, 2019; Published: September 23, 2019

DOI: 10.31080/ASMI.2019.02.0381

\begin{abstract}
Periodontitis is a multifactorial disease, one of the responsible factors include Bacteria present in the oral cavity. Bacteria like all other organism are dependent on the environment for their survival and are influenced by various Host as well as environmental factors for their sustenance. Some of the major environmental factors include nutrition, presence of oxygen along with $\mathrm{pH}$ which dictate the nature of the bacteria present as certain bacteria thrive better in acidic environments while others in Alkali environments. In medicine sutures are extensively used with an aim of post surgical wound edge approximation or wound healing by primary union. However $\mathrm{pH}$ is one factor which is constantly changing in the oral cavity due to dietary intake or systemic factors or local accumulation of pus.

Considering all these factors the rationale of this study was to assess the biohostability of different suture material such as Silk, Vicryl and Chromic gut when $\mathrm{pH}$ was changed to 5.6,7 keeping all other environmental factors a constant.

The results obtained suggested that $\mathrm{pH}$ plays a vital rile in the Biohostability of suture material as an Acidic $\mathrm{pH}$ of 5 had higher colony forming units seen as compared to the other 2 groups.
\end{abstract}

Keywords: Sutures; pH; Vicryl; Chromic Gut; Silk; Biohostability

\section{Introduction}

Periodontology has considered Bacteria as the sole cause for infection for centuries and has attributed several years in the study different Microflora that comprise the oral Microbiome and the extent of destruction caused by each individual bacteria or bacterial species [1]. Newer research over the past 2 decades has provided sufficient evidence of a possible role of both fungi $[2,3]$ and $\mathrm{Vi}$ ruses $[4,5]$ as being etiological agents in the pathogenesis of the Periodontal disease. Despite all these advances, Periodontitis till date has been established as a multifactorial disease with multiple predisposing factors and etiological agents as the severity, pattern and progression of this disease could not be solely explained by the amount of plaque present [6]. This led to an alternative approach in 1999, where alterations of the Host response towards various pathogens was targeted instead of targetting specific organisms itself [7-9].

The extensive study of different bacteria had suggested various environmental as well as host related factors that affect the growth of Bacteria at specific site. They are Temperature, Extent of Oxygen available in the environment, nutrition, and also $\mathrm{pH}$. $\mathrm{pH}$ is an abbreviation for potential of Hydrogen suggesting the acidic or alkaline nature of a site and the possible change in such a nature which would greatly affect bacteria growth [10]. There are certain
Bacteria which favour an acidic medium while other prefer an alkali medium optimal for growth. The rationale of the following study was to asses if $\mathrm{pH}$ played any significant role in affecting the bacterial growth on different sutures if the Microflora cultured in these materials was standardised.

\section{Material and Methods}

Aim

- To assess biohostability of different suture materials at different $\mathrm{pH}$.

- To quantify the difference in Biohostability using visual click counter method.

The following study was conducting using the help of the Department of Microbiology in a Chennai Dental College.

Three of the most commonly used clinical sutures were selected namely 4-0 perma Silk, 4-0 Vicryl and 4-0 Chromic gut all of which were made by Ethicon manufactured in India by Johnson and Johnson Pvt. They were then standardised by cutting them into 9 equal lengths measuring 1 inch or 2.5 centimetres. These suture materials were then sent for Autoclaving using "Life Stericare $10 \mathrm{~L}$ dental autoclave" manufactured by Life Dental and Clinical Autoclaves Pvt. at the specification of 115 degrees Celsius for 15 minutes at 15 bar pressure. 
To make a medium for bacterial growth which would not change in $\mathrm{pH}, 210 \mathrm{ml}$ of $10 \%$ Phosphate buffer Saline commercailly available as a $500 \mathrm{ml}$ container from Lonza chemical India Pvt. was divided into 3 separate sterile conical flasks obtained from Amar Pvt labelled A, B, C. The pH of A was set at 5 using a combination of both Hydrochloric acid and Sodium hydroxide obtained from the Microbiology and Pathology Department of Saveetha Dental College which was then titrated individually drop by drop till the $\mathrm{pH}$ of 5 was attained without overshooting it and matched using $\mathrm{pH}$ paper made by Merck. Using a similar procedure the $\mathrm{pH}$ of $\mathrm{B}$ was set till 6 and $\mathrm{pH}$ of $\mathrm{C}$ was set till 7. The following labelled Conical flasks was autoclaved at the previously mentioned temperature, time and pressure specifications. After sterilisation $5 \mathrm{ml}$ was collected from the conical flasks labelled A,B and C and transferred into a sterile plastic contained with a lid provided by Amar Pvt. To standardise the Microbiological load exposed 0.5 Mcfarland units Lactobacillus Subculture was added equally to all 3 containers A,B, and C. Sterile tweezers were used to transfer 3 sutures lengths of 1 inch each from their autoclaved packaging to all three containers such that there were 3 silk sutures, 3 vicryl sutures and 3 chromic gut sutures in container A,B and C. The containers were then closed and placed in an SSU Bacteriological Incubator at 35 degrees celsius for a period of 4 hours. After 4 hours, using another sterile tweezer each individual sutures was transferred from their containers to a holding solution of $1 \mathrm{ml}$ sterile Normal Saline commercially available as $100 \mathrm{ml}$ mini bottles of $0.9 \%$ Sodium Chloride manufactured by the Baxter corporation in a sterile Cuvvette labelled from 1 to 27 while carefully recording which number corresponded to which suture kept at which $\mathrm{pH}$ and shaken thoroughly. Using a $0.5 \mathrm{ml} \mathrm{Ep-}$ pendorf tube attached to individual tips normal saline from each cuvvette was transferred to a separate nutrient agar coated plates labelled $\mathrm{A}, \mathrm{B}$ and $\mathrm{C}$ (as per $\mathrm{pH}$ ) which were then agitated and the spread plate method was used to uniformly distribute the suspension across the entire dimension of the plate using metal loops sterilised by red hot flame sterilisation and Incubated overnight for the specifications mentioned above.

The subsequent morning the plates were examined to rule out contamination by any other form of Bacterial Colonies and divided into 4 individual quadrants using a permanent marker and the number of individual Colony forming units was counted for all the 27 plates. The average of the values were recorded and results were mentioned as follows.

\section{Results}

The following results were obtained from the above mentioned study are tabulated below based on the average colony forming units of suture materials at different $\mathrm{pH}$ for all three sets of sutures observed by two different Observers to rule out any form of Bias, both observers used visual examination method using a pen and Click counter method to quantify observations.

\begin{tabular}{|l|c|c|c|}
\hline Different pH & VICRYL (CFU) & SILK (CFU) & $\begin{array}{c}\text { CHROMIC } \\
\text { GUT (CFU) }\end{array}$ \\
\hline pH 5 & 12 & 27 & 24 \\
\hline pH 6 & 14 & 10 & 5 \\
\hline pH 7 & 3 & 1 & 16 \\
\hline
\end{tabular}

Table 1: Average value obtained from three different sets of same suture materials.

\section{Discussion}

Sutures are routinely used in clinical practice of periodontology as a means of closing surgical sites post surgical intervention allowing healing to occurs by either approximation or primary union of gapping wound edges. Initially they were seen as the only means to close surgical sites however newer products such as Antibiotic coated sutures seem to reduce bacterial load and provide beneficial effects for short periods of time [11]. As an alternative to conventional suturing techniques Cyanoacrylate glues [12,13] and surgical staples have also gained popularity in performing similar functions. Fibrin based surgical glues currently seem to be the pinnacle of technology in terms of surgical site closure preferred over Cyanoacrylate glues [14].

Considering how often sutures, surgical glues and staples have been used in the human body their biocompatability and physical properties have extensively been studied, however all these studies were carried out in an environment where $\mathrm{pH}$ was kept a constant. The Oral cavity or the mirror of the body is constantly subject to extreme temperatures as well as drastic change in $\mathrm{pH}$ in a matter of seconds for ingestion of certain type of food groups such as Carbonated drinks or Lemon juice $\mathrm{pH}$ 2.6, orange juice 3.3, milk 6.8 . The presence of focal infection at a site with accumulation of pus alters $\mathrm{pH}$, not forgetting certain systemic conditions such as Acid Reflex also drastically alter the pH.15,16 Several studies were performed which evaluated the change in physical properties of suture material as $\mathrm{pH}$ was changed finding both clinical and statistical significance $[17,18]$. The possibility of $\mathrm{pH}$ significantly altering the physical properties such as tensile stress and modulous of elasticity of suture material lead to the rationale that perhaps it could also alter their biological or microbial properties.

The three $\mathrm{pH}$ selected in this study were chosen based on critical pH of Enamel, normal pH of the Oral cavity, changes in $\mathrm{pH}$ which could be expected as a result of accumulation of pus from any infection as well as changes which occurs due to ingestion of certain types of food such as soft drinks and citrus juices. Despite all these above mentioned factors which can change $\mathrm{pH}$, the human body functions in a balanced manner also known as homeostasis, which is capable of bringing the $\mathrm{pH}$ of the mouth back to the physiological $\mathrm{pH}$. Thus the $\mathrm{pH}$ selected for the above study was physiological $\mathrm{pH}$ of 7 along with a $\mathrm{pH}$ of 5,6.Standardisation and how the entire 
protocol was formulated and followed was carefully planned and rationed out to ensure best results including 1 Mcfarland units of bacterial suspension instead of in-dependant mouth swabs as each successive mouth swab may vary in Bacteria present which could cause bias. No contamination was seen as a high grade of sterilisation protocol was followed.

Another important feature which became apparent after the entire study was the nature of chromic gut, which is preserved in an alcohol based medium provided by the manufacturer itself. When this seal was broken to standardise the lengths of the suture to be used for the study itself, there would have been some change in the physical properties of Chromic gut causing it to shrink in size. This could be one of the explanations for the unusual results and observations obtained for chromic gut however in previous studies which compared physical properties did not consider the removal from alcohol based medium relevant enough.

\section{Conclusion}

In conclusion $\mathrm{pH}$ plays a vital rile in the Biohostability of suture material as evidence above suggests that in an Acidic $\mathrm{pH}$ of 5 there were more colony forming units seen as compared to the other 2 groups. A possible explanation behind this could be because of the higher affinity of Lactobacillus colonies towards an acidic $\mathrm{pH}$ which favoured their growth in all three sutures irrelevant of their nature.

\section{Bibliography}

1. Socransky SS., et al. "New Concepts of Destructive Periodontal Disease". Journal of Clinical Periodontology 11. 1 (1984): 21-32.

2. B. Urzúa G., et al. "Yeast Diversity in the Oral Microbiota of Subjects with Periodontitis: Candida Albicans and Candida Dubliniensis Colonize the Periodontal Pockets | Medical Mycology | Oxford Academic".

3. Jørgen Slots., et al. "Yeasts, Enteric Rods and Pseudomonads in the Subgingival Flora of Severe Adult Periodontitis - Slots - 1988 - Oral Microbiology and Immunology - Wiley Online Library".

4. Slots Jørgen. "Herpesviruses, the Missing Link between Gingivitis and Periodontitis?" Journal of the International Academy of Periodontology 6.4 (2004): 113-119.

5. Slots, Jorgen. "Oral Viral Infections of Adults". Periodontology 49 (2009): 60-86.

6. Ryan ME and Preshaw PM. "Host Modulation". In: Newman MG, Takei HH, Klollevold PR, Carranza FA, editors. Carranza's Clinical Periodontology 11th ed. India: Saunders; (2012): 275-280.

7. Golub LM., et al. "Host Modulation with Tetracyclines and Their Chemically Modified Analogues". Current Opinion in Dentistry 2 (1992): 80-90.
8. Williams RC. "Periodontal Disease". The New England Journal of Medicine 322.6 (1990): 373-82.

9. PM Preshaw. "Host Response Modulation in Periodontics". 48 (2008): 92-110.

10. 10-Yeaw and Ralph Cady. "The Effect of PH on the Growth of Bacteria in Urine121Read before American Urological Association, Buffalo, NY., on June 26, 1940". The Journal of Urology 44. 5 (1940): 699-712.

11. M Indumathi and Dr. Sivakumar. "Application of antibacterial suture material in Oral nd Maxillofacial surgery" Drug invention 12.1 (2019).

12. McGraw VA and Caffesse RG. "Cyanoacrylates in Periodontics". Journal of the Western Society of Periodontology/Periodontal abstracts 26 (1978): 4-13.

13. Torumi DM and O'Grady K. "Surgical tissue adhesives in otolaryngology-head and neck surgery". Otolaryngologic Clinics of North America 27 (1994): 203-209.

14. Jathal Bimal., et al. "Use of Fibrin Glue in Periodontal Flap Surgery". Journal of Indian Society of Periodontology 12.1 (2008): 21-25.

15. Cheng., et al. "Dental Erosion and Severe Tooth Decay Related to Soft Drinks: A Case Report and Literature Review". Journal of Zhejiang University Science. B 10. 5 (2009): 395.

16. Nekoofar, et al. "PH of Pus Collected from Periapical Abscesses". International Endodontic Journal 42 (2009): 534-538.

17. Tomihata., et al. "The PH Dependence of Monofilament Sutures on Hydrolytic Degradation". Journal of Biomedical Materials Research 58 (2001): 511-518.

18. Harshinee Chandrasekhar., et al. "Comparison of Influence of Vicryl and Silk Suture Materials on Wound Healing After Third Molar Surgery- A Review". Journal of Pharmaceutical Science and Research 9.12 (2017).

\section{Volume 2 Issue 10 October 2019}

(C) All rights are reserved by Siddharth Narayan. 